\title{
Chinese Academy of Sciences
}

For 65years, the Chinese Academy of Sciences has been a rich source

ARTICLE COUNT (AC): 2,661

of technological innovation, scientific discovery and aspiring minds. Making the leap from a regional to a global leader, researchers are taking the intellectual powerhouse to soaring new heights.

FRACTIONAL COUNT (FC): 1,351

WEIGHTED FRACTIONAL COUNT

(WFC): 1,209
$\mathrm{T}$ The Chinese Academy of Sciences (CAS) is the world's largest scientific organization, with about 48,500 researchers in 114 directly controlled institutes spread across the country. Its annual budget for 2013 was US $\$ 5.4$ billion. Over the last 65 years, CAS has made many important discoveries and technological advances across diverse fields, including making the first synthetic insulin from bovine sources (1965); building China's first particle accelerator, the Beijing Electron-Positron Collider (1984); and the discovery of iron-based high-temperature superconductors (2008).

There are 49 CAS institutes based in Beijing, including the Institute of Chemistry (ICCAS), the Institute of Physics (IOP), the Institute of Atmospheric Physics (IAP) and the Institute of Vertebrate Paleontology and Paleoanthropology (IVPP). Key institutes outside Beijing include the Changchun Institute of Applied Chemistry (CIAC), Dalian Institute of Chemical Physics (DICP), Shanghai Institute of Organic Chemistry (SIOC), Shanghai Institutes for Biological Sciences (SIBS) and the Purple Mountain Observatory (PMO) - the latter being based in Nanjing (page S66).

In 2013, CAS published 2,661 articles (WFC = 1,209 ) in the 68 high-quality journals that comprise the Nature Index. It has a larger output in the index than all the other research institutions worldwide - and in fact has a higher WFC than many scientifically advanced countries including Spain, Switzerland and South Korea.

CAS is also a regular contributor to Nature and Science, having published 54 articles (WFC $=18.6$ ) in these two top journals in 2013 (see 'Nature and Science breakdown'). By WFC this represents one-third of China's total contribution to Nature and Science, reflecting the organization's strength in basic research.

\section{"WE CAN NOW DETERMINE THE ORIGIN OF DINOSAURS AND PROVIDE ANSWERS TO THEIR EVOLUTIONARY HISTORY."}

Here we look at the leading institutes in the four broad subject areas (see 'CAS subject split'), as well as in the Nature and Science category.

ICCAS, founded in 1956, is the leading CAS institute overall by WFC, and dominates the competitive field of chemistry. In 2013, it published 244 articles $(\mathrm{WFC}=124.7)$ across a wide range of subfields including analytical chemistry, materials chemistry, organic chemistry and physical chemistry. Top contributor is Lanqun Mao from the laboratory of analytical chemistry for life sciences, who co-authored seven articles $(\mathrm{WFC}=4.9)$ on electrochemical biosensors. $\mathrm{He}$ is closely followed by Huimin $\mathrm{Ma}$, from the same laboratory, who wrote four articles with ICCAS colleagues $(\mathrm{WFC}=4)$ on fluorescent probes.

Another major contributor at ICCAS is Song Ye from the molecular recognition and selective synthesis laboratory. In 2013, Ye led four articles $(\mathrm{WFC}=3.9)$ on the development of novel catalysts for use in asymmetric synthesis, all in the journal Angewandte Chemie International Edition. Ye explains that in normal asymmetric synthesis of pharmaceuticals using metal catalysts, the catalyst must be removed from solution in a post-treatment process to prevent toxic metals from getting into the final product. "We have discovered an organocatalyst that eliminates the need for this step," he says.

Yuguo Guo from the Key Laboratory of Molecular Nanostructures and Nanotechnology is another prolific author. Guo co-authored three articles $(\mathrm{WFC}=3$ ) on lithium-ion batteries in 2013. In particular, his article titled "Binding $\mathrm{SnO}_{2}$ nanocrystals in nitrogen-doped graphene sheets as anode materials for lithiumion batteries", published in Advanced Materials, was listed as one of China's most influential

\section{CAS ANALYSIS}

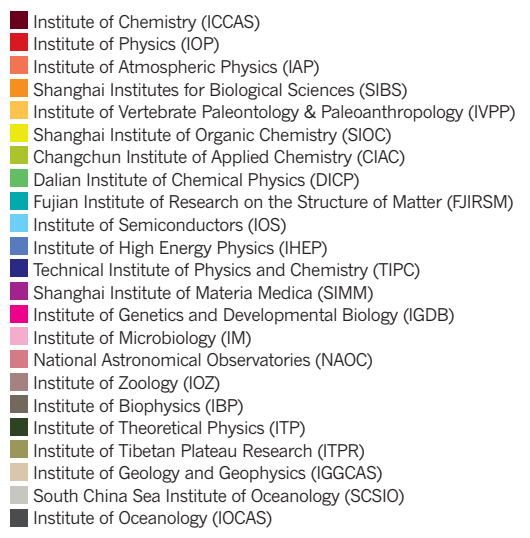

Researcher efficiency

Each ICCAS researcher contributed just over

1 point of WFC to their institute.

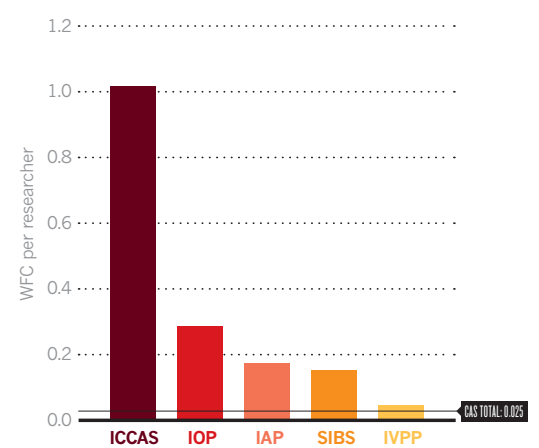

Contributing institutions

Chemistry leads overall, but there is no dominant institute of those in the Nature Index.

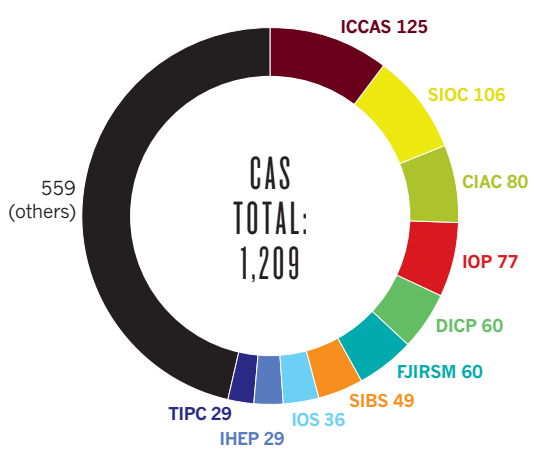


papers in 2013 by the Institute of Scientific and Technical Information of China.

Scientists from ICCAS are also among the most efficient, index data show. There are 123 researchers who have contributed to a WFC of 125: one of the highest ratios we have calculated for any institution (see 'Researcher efficiency').

IOP, one of the oldest CAS institutes and among the top five by WFC in the index, represents the largest contributing institute in the physical sciences. In 2013, the 64-year-old establishment published 172 articles (WFC = 77.2) in the index, with a focus on condensed matter physics. Xucun Ma from the State Key Laboratory for Surface Physics is the most active contributor. She worked on ten articles (WFC =4.7) - including one in Science - on hightemperature superconductors.

Other major contributors at the IOP include Baogen Shen from the Beijing National Laboratory for Condensed Matter Physics. Shen led six articles $(\mathrm{WFC}=4.1$ ) on magnetocaloric effect the heating or cooling of materials by the application of magnetic fields. In his immediate wake is Yongsheng $\mathrm{Hu}$, who produced four articles, all co-authored by IOP scientists $(\mathrm{WFC}=4)$, on the development of electrode materials for use in lithium-ion batteries - two of which, in Nature Communications, were listed among China's most influential papers. Hu's discovery concerned a new class of electrolytes that improve the performance of conventional lithium-ion batteries. "The material also improves the battery life and stability by preventing the formation of crystals," he explains.

SIBS is the dominant institute by WFC in the life sciences - and the second largest contributing institute overall of those based in Shanghai (topped only by SIOC). Founded in 1999, SIBS published 111 articles (WFC $=49.3$ ) in 2013, covering a wide range of subfields including cell biology, molecular biology, neurobiology and structural biology. When it comes to publications in Nature and Science, SIBS also has the highest WFC of any CAS institute - and

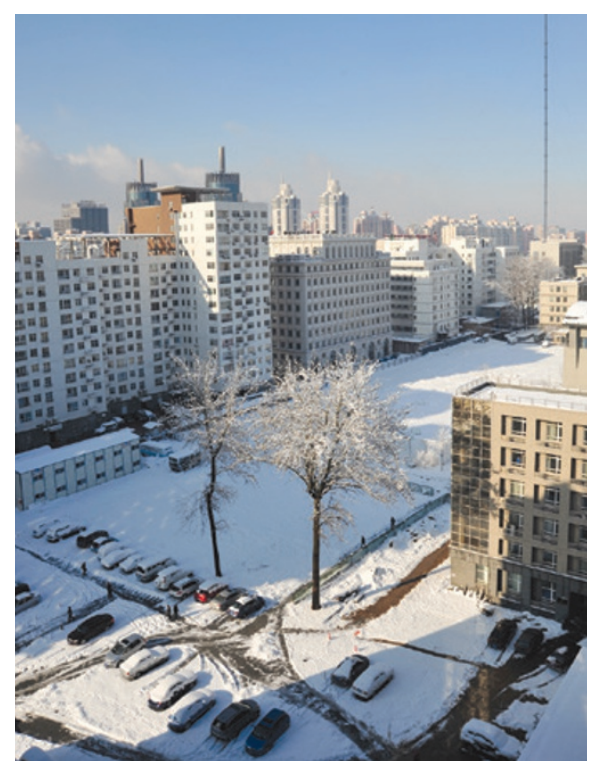

The Institute of Atmospheric Physics in Beijing

is third overall for China - for its eight articles (WFC = 3.8) in these two prestigious journals. In particular, plant biologist Peng Zhang from the Institute of Plant Physiology and Ecology led one article comprised entirely of SIBS researchers $(\mathrm{WFC}=1)$ in Nature; in this paper, they solved the structure of a folate energy-coupling factor transporter protein, which is involved in vitamin and micronutrient uptake in prokaryotes.

Other major contributors at SIBS include Xinyuan Liu $(\mathrm{WFC}=1.5)$ and Guoliang Xu $(\mathrm{WFC}=0.9)$ from the Institute of Biochemistry and Cell Biology. Liu co-wrote two articles $(\mathrm{WFC}=1.5)$ on the Hippo signalling pathway, which plays an important role in the regulation of cell proliferation and controlled cell death. Xu co-authored three articles $(\mathrm{WFC}=0.9)-$ including one in the journal Cell - on cell reprogramming and neurogenesis.

IAP is by far the biggest contributing institute in earth and environmental sciences. The 48-year-old establishment has 44 articles
(WFC $=18$ ) in the index, most of which were published in the Journal of Geophysical Research: Atmospheres. This is not an area of strength for CAS in the index in general, nor indeed for Chinese science overall. In fact, IAP accounts for one-quarter of CAS's WFC in earth and environmental sciences. There are three major contributors at IAP researching vastly different areas: Tao Wang, who published two articles $(\mathrm{WFC}=1.5)$ on the palaeoclimate; Zhenghui Xie, who published two articles $(\mathrm{WFC}=1.2)$ on satellite measurements of surface solar radiation; and Tianjun Zhou, who published two articles $(\mathrm{WFC}=1.1)$ on long-term changes in the troposphere.

IVPP is not only the largest contributing institute by WFC in palaeontology, but also the largest contributor by percentage of WFC to Nature and Science. In 2013, half IVPP's articles were in these two journals, giving it the highest ratio for CAS institutes. However, its total output is fairly modest: IVPP has only 14 articles (WFC $=4.2$ ) in the index. Xing Xu from the department of paleoichthyology and paleoherpetology is the most active writer at IVPP, having produced three articles (WFC = 1.1) - including one in Science - on fleas from the cretaceous period, and on early dinosaurs.

"The traditional method for dinosaur classification is through rigorous analysis of unique characteristics and taxonomy," says Xu. "With advances in genetics, developmental biology and bone histology, we can accurately determine the origin of dinosaurs and provide confirmative answers to their evolutionary history."

From the same department, Zhonghe Zhou is the second most active contributor at IVPP, having co-authored two articles $(\mathrm{WFC}=0.9)$ — including one in Nature - on the evolution of early birds. "We discovered the fossils of three early birds, all carrying one functional ovary on the left side of their body," says Zhou. "This suggests that the right ovary was lost in the dinosaur-avian transition and sheds new light on the early evolution of modern avian reproduction."

\section{CAS ANALYSIS}

Nature and Science breakdown

Life sciences institutes contribute most to papers in these two journals.

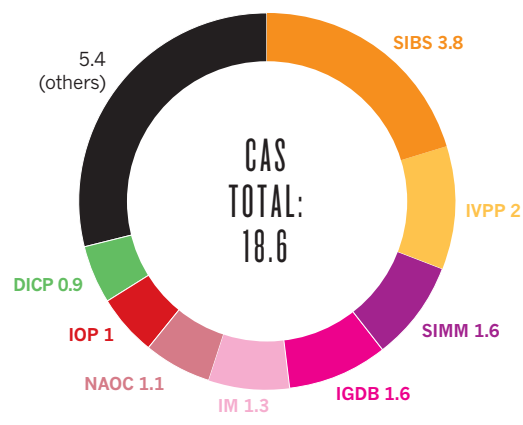

Nature and Science ratio

As a proportion of total WFC, life sciences command a dominant share in these two journals.

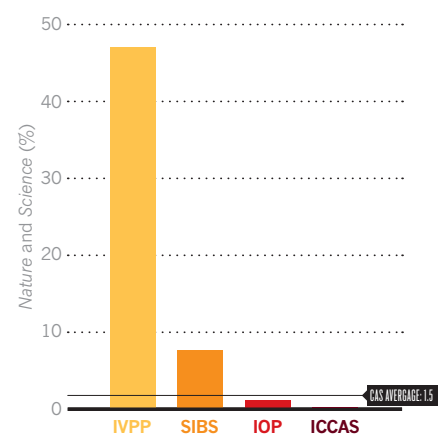

CAS subject split

The leading institutions by proportion of WFC for each of the four subject areas.

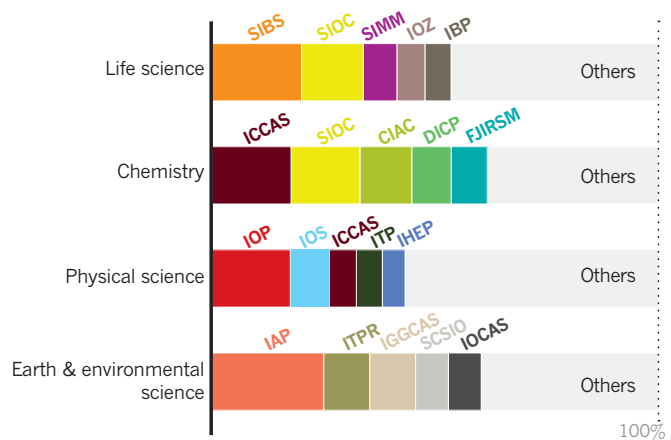

